\title{
EFFECT OF MAXIMAL EXERCISE ON THE AUTONOMIC CONTROL OF HEART RATE DURING ORTHOSTATIC STRESS
}

\author{
J.E. Davis, W.R. Pourcho, and C.M. Thomas
}

Dept. of Exercise and Health Science, Alma College, Alma, Michigan, 48801 USA

\section{INTRODUCTION}

Hypotension is a commonly observed phenomenon after acute maximal exercise. In some instances this can lead to lightheadedness and even syncope (1). The mechanism underlying this phenomenon has yet to be determined. Some studies have suggested that the baroreflex mechanism is altered and point to decreases in peripheral vascular resistance after a single bout of maximal exercise (2). It is possible that changes in the control of heart rate after exercise in some way contribute to the hypotension.

One non-invasive way of determining autonomic input on the control of heart rate is the assessment of beat to beat changes in heart rate dynamics (3). By quantifying heart rate dynamics with spectral analysis, sympathetic and parasympathetic control of cardiac function can be assessed (4). This technique has been used to investigate alterations in cardiovascular control for a wide variety of environmental stresses including altitude (5) and bed rest (6).

In this study, spectral analysis was used to examine the effects of a maximal bout of aerobic exercise on the autonomic nervous system's control of heart rate during an orthostatic challenge. We hypothesized that the maximal exercise would reduce orthostatic tolerance and alter the autonomic control of heart rate. Altered autonomicfunctioning may help to explain syncopal episodes following exercise.

\section{MATERIALS and METHODS}

Subjects: Five healthy subjects participated in the study. All subjects were of average fitness level with a mean age of $20.8 \pm .20$. Each subject gave informed consent after receiving a complete description of the procedures and potential risks of the study.

Experimental Protocol: Each subject participated in two orthostatic tolerance tests, a control test $(\mathrm{C})$ with no prior exercise and a test following a Bruce maximal graded exercise test (GXT). Lower body negative pressure (LBNP) was used to determine orthostatic tolerance. A progressive LBNP protocol began with a 5 minute pre-test period with the subject supine in a specially designed LBNP box without negative pressure. 
Pressure began at $-20 \mathrm{mmHg}$ and increased by $-10 \mathrm{mmHg}$ every 3 minutes to the onset of presyncopal symptoms. At the onset of these symptoms the negative pressure was terminated and an eight minute post-test followed with the subject supine in the LBNP box. Orthostatic tolerance is expressed as a cumulative stress index (sum of minutes at each stage $\mathrm{x}$ pressure for each stage).

Heart rate was determined with a standard 3-lead electrocardiograph using a Biopacq MP-100 data acquisition system (Goleta, CA). A time series of heart rate was then determined for further analysis. One non-invasive way of examining the autonomic control of cardiac function is through the use of power spectral analysis of heart rate fluctuations $(3,4)$. Spectral analysis in this study included a fast Fourier transformation of the time series plot into a power spectrum. Integration of the area under the curve in the high frequency band, low frequency band, and total spectrum was performed to quantify the area in each frequency domain. In order to quantify the influences of both branches of the autonomic nervous system, the integrated area under the curve is recorded for three areas: the $\mathrm{P}_{\mathrm{H}}, \mathrm{P}_{\mathrm{L}}$, and the total power. Oscillations in the high frequency $\left(\mathrm{P}_{\mathrm{H}}\right)$ band $(.15$ to $.50 \mathrm{~Hz})$ of the power spectrum are influenced by parasympathetic (PNS) input to the heart, and oscillations in the low frequency $\left(\mathrm{P}_{\mathrm{L}}\right)$ band $(0$ to $.15 \mathrm{~Hz})$ are influenced by both PNS and sympathetic (SNS) $(3,4)$. The ratio of the integrated power of $P_{L} / P_{H}$ corresponds to the SNS input to the heart; the ratio of integrated power of $P_{y} / P_{T}$ (total area of the spectrum) corresponds to the PNS input to the heart.

A one-way analysis of variance for repeated measures was used to determine statistical significance. Post-hoc analyses were performed using a Scheffe's test. The .05 level was established as the criterion for acceptance. All data in subsequent displays are expressed as mean $\pm \mathrm{SE}$.

\section{RESULTS}

Orthostatic Tolerance: There was a significant $(\mathrm{P}<0.05)$ decrease in orthostatic tolerance after maximal exercise $(G X T)$ compared to the control test. Tolerance, in cumulative stress units, decreased from $844.7(592.2 \mathrm{~mm}$. min) in the control condition to 706.0 ( $\pm 46.7 \mathrm{~mm}$. $\mathrm{min}$ ) following the graded exercise test.

Heart Rate: Heart rate significantly increased $(\mathrm{P}<0.05)$ during LBNP in both tests (Figure 1). In addition, heart rates were higher before and at each stage of the LBNP following the graded exercise test as compared to the control LBNP. Heart rate decreased below resting level after LBNP for both the control and post-graded exercise conditions.

$\boldsymbol{P}_{L} / \boldsymbol{P}_{H}$ Ratio: The $\mathrm{P}_{\mathrm{L}} / \mathrm{P}_{\mathrm{H}}$ ratio results are shown in Figure 2. $\mathrm{P}_{\mathrm{L}} / \mathrm{P}_{\mathrm{H}}$ ratio was significantly greater $(\mathrm{P}<0.05)$ at the start of the LBNP after the graded exercise test 


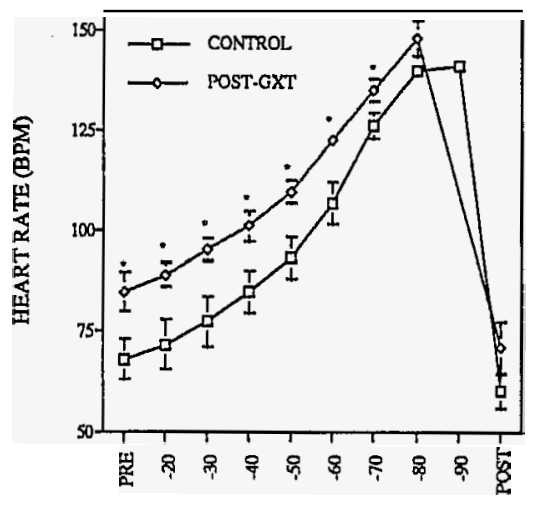

STAGES OF LBNP

Figure 1. Heart rate as a function of LBNP stage for the control and post-GXT condition. Values expressed are means \pm 1 SE. * indicates a significant difference from control $(\mathrm{P}<0.05)$.

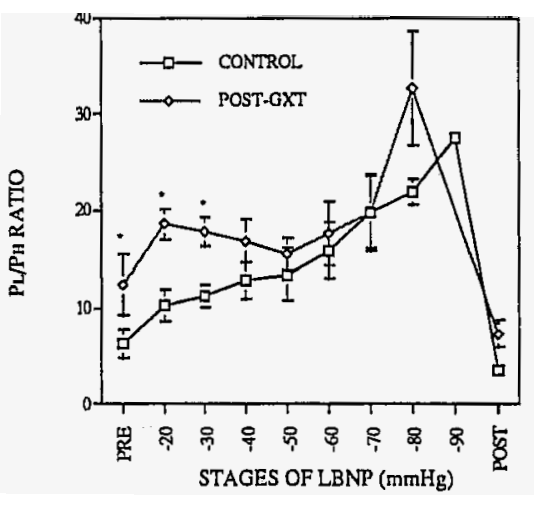

Figure 2. $P_{L} / P_{H}$ ratio as a function of LBNP stage for the control and post-GXT condition. Values expressed are means \pm 1SE. * indicates a significant difference from control $(\mathrm{P}<0.05)$.

(12.41 \pm 3.18$)$ as compared to the control test $(6.36 \pm 1.49)$. This is indicative of greater sympathetic influence on resting heart rate after exercise. The $\mathrm{P}_{\mathrm{L}} / \mathrm{P}_{\mathrm{H}}$ ratio increased during both LBNP tests. However, the $\mathrm{P}_{\mathrm{L}} / \mathrm{P}_{\mathrm{H}}$ was significantly greater during LBNP after the graded exercise test than the control condition through the $-30 \mathrm{mmHg}$ stage of LBNP. This reflects greater sympathetic activity during lower levels of LBNP after graded maximal exercise.

$P_{H} P_{T}$ ratio: The $\mathrm{P}_{\mathrm{H}} / \mathrm{P}_{\mathrm{T}}$ ratio was lower $(\mathrm{P}<0.05)$ at the start of the GXT LBNP test $(.110 \pm .040)$ as compared to the $C$ test $(.164 \pm .039)$ and remained depressed until the $-30 \mathrm{mmHg}$ stage of LBNP (Figure 3). This indicates that parasympathetic activity

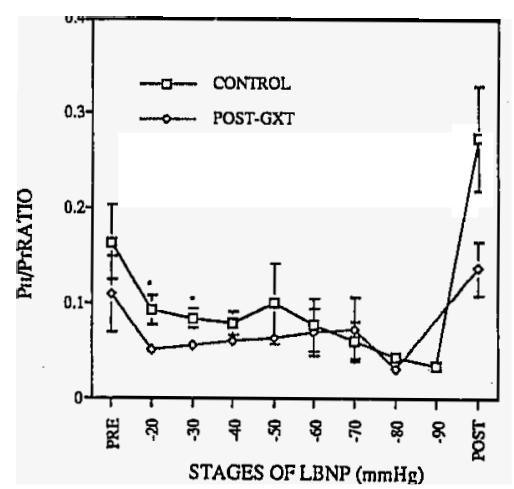

Figure 3. $\mathrm{P}_{\mathrm{H}} / \mathrm{P}_{\mathrm{T}}$ ratio as a function of LBNP stage for the control and post-GXT condition. Values expressed are means \pm 1SE. ${ }^{*}$ indicates a significant difference from $\operatorname{control}(P<0.05)$. 
at rest and during low levels of LBNP is reduced after maximal exercise relative to the control condition. During the post-test the C LBNP produced a higher $P_{H} / P_{T}$ ratio $(.273 \pm .056)$ than the GXTLBNP $(.137 \pm .028)$.

\section{CONCLUSIONS}

The major finding of this study is that the autonomic control of heart rate during orthostatic stress is altered after maximal aerobic exercise. We found higher heart rates after maximal exercise at rest and during a progressive LBNP test. These higher heart rates are a result of greater sympathetic and less parasympathetic stimulation of the heart. Sympathetic nervous system input on the heart after maximal exercise was greater and parasympathetic input was reduced relative to a control lower body negative pressure at lower levels of LBNP $(\leq 30$ $\mathrm{mmHg}$ ).

It is possible that the altered heart rates and control mechanisms after exercise are a result of changes in baroreceptor function. Halliwell et al. (2) have reported augmented heart rate gain after moderate intensity exercise. They proposed that this restrains post-exercise hypotension and partially offsets the enhanced vasodilation observed after exercise. It also is possible that the altered autonomic control of heart rate observed in this study in some way contributes to the syncopal episodes that have been observed following maximal exercise.

\section{REFERENCES}

1. Holhausen, L.M., Noakes, T.D., Kroning, B., DeKlerk, M., Roberts, M., Emsley, R. 1994, Clinical and biochemical characteristics of collapsed ultramarathon runners. Med. Sci. Sports. Exerc. 26: 1095-1101.

2. Halliwell, J.R., Taylor, J.A., Hartwig, T.D. and Eckberg, D.L. 1996, Augmented baroreflex heart rate gain after moderate-intensity, dynamic exercise. Am. J. Physiol. 270 (Reg.Int. Comp. Physiol. 39): R420-R426.

3. Akselrod, S., Gordon, D., Ubel, F.A., Shannon, D.C., Barger, A.C. and Cohen, R.J. 1981, Power Spectrum Analysis of Heart Rate Fluctuation: A Quantitative Probe of Beat-to-Beat Cardiovascular Control. Science 213: 220-222.

4. Pomeranz, B., Macaulay, R.J., Caudill, M.A., Kutz, I., Adam, D., Gordon, D., Kilborn, K.M., Barger, A.C., Shannon, D.C., Cohen, R.J. and Benson, H. Assessment of autonomic function in humans by heart rate spectral analysis. Am. J. Physiol 248 (Heart Circ. Physiol. 17): H151-H153.

5. Hughson, R.L., Yamamoto, R.E., McCullough, J.R., Sutton, J.R. and Reeves, J.T.1994, Sympathetic and parasympathetic indicators of heart rate control at altitude studied by spectral analysis. J. Appl. Physiol. 77(6): 2537-2542.

6. Goldberger, A.L., Mietus, J.E., Rigney, D.R., Wood, M.L. and Fortney, S.M. 1994, Effects of head-down bed rest on complex heart rate variability; response to LBNP testing. J. Appl. Physiol. 77(6): 2863-2869. 